\title{
Effects of Isolated and Mesenchymal Stem Cells- Loaded Chitosan on Repair of Cranial Critical-Size Defect in Rats
}

\author{
Caio Almeida Batista de Oliveira ${ }^{1,2}$, Carla Roberta Tim ${ }^{3 *}$, Cintia Cristina Santi Martignago ${ }^{4}$, Lia \\ Mara Grosso Neves ${ }^{4}$, Livia Assis ${ }^{3}$, Genoveva Lourdes Flores Luna ${ }^{5}$, Nivaldo Antonio Parizotto ${ }^{4}$ \\ and Karina Nogueira Zambone Pinto Rossi ${ }^{1}$ \\ ${ }^{1}$ Department of Microbiology and Pathology, Federal University of São Carlos, Brazil \\ ${ }^{2}$ Department of Genetics and Evolution, Federal University of São Carlos, Brazil \\ ${ }^{3}$ Department of Biomedical Engineering, University Brazil, Brazil \\ ${ }^{4}$ Department of Physiotherapy, Federal University of São Carlos, Brazil \\ ${ }^{5}$ Department of Medicine, Federal University of São Carlos, Brazil. \\ *Corresponding author: Carla Roberta Tim, Department of Biomedical Engineering, University Brazil, Brazil
}

\begin{abstract}
ARTICLE INFO
Received: 幽 April 15, 2019

Published: April 23, 2019

Citation: Caio Almeida B O, Carla Roberta T, Cintia Cristina S M, Lia Mara G N, Livia A, et al. Effects of Isolated and Mesenchymal Stem Cells-Loaded Chitosan on Repair of Cranial Critical-Size Defect in Rats. Biomed J Sci \& Tech Res 17(2)-2019. BJSTR. MS.ID.002986.
\end{abstract}

Keywords: Biomaterials; Chitosan; Bone; Tissue Engineering; Bone Repair

\section{ABSTRACT}

Chitosan is used in different forms and with varied objectives, among which the applicability in hydrogel form has been brought out, for treatment of joint and bone diseases and traumas, with or without cell loading. Thus, it was the aim of this study to evaluate the efficacy of chitosan hydrogel implant, associated or not to Mesenchymal Stem Cells (MSCs), in repair of critical sized bone defects induced on rats. In vitro tests involved hydrogel degradation and MSCs' viability tests in contact with the hydrogels. In vivo tests used thirty male Wistar rats, randomly distributed in three groups, each with 10 animals (control group without treatment; group treated with chitosan; group treated with MSCs-loaded chitosan). After 21 days, animals were euthanized by anaesthetic lethal dose. Neither of in vitro tests (degradation and cell viability) presented significant difference among groups. In vivo results showed a great number of cells inside defect region in qualitative histological slides of treated groups, apart from biomaterial residues.

The morphometric analysis showed a greater presence, statistically significant, of collagen in the group treated with isolated chitosan when compared to control. Immunohistochemical results did not present significant difference regarding presence of Runt-Related Transcription Factor 2 (RUNX-2) and Osteocalcin (OC) factors. However, it showed a higher immunostaining of cyclooxygenase 2 (COX-2) in the group treated with isolated chitosan in relation to control group. We concluded the chitosan hydrogel is capable to help the bone repair process, with potential applications in tissue engineering and regenerative medicine.

\section{Introduction}

The process of bone repair consists in a complex sequence of events involving several molecular mediators and cellular types. Among these cells, there are the Mesenchymal Stem Cells (MSCs)described firstly by Friedenstein et al. [1] -, which are capable to divide but remaining undifferentiated. Lately, they can differentiate into mesodermal cell lineages, as osteoblasts, which are an essential part of bone formation and maintenance; in addition, they are also capable to modulate inflammatory response, cooperating with the repair process [2-4]. When a lesion occurs, a provisional extracellular matrix is formed, recruiting and activating different cells, which are responsible to release the first signalling molecules of the inflammatory response [5,6]. Among these mediators, some play an important role during the process, as the enzyme Cyclooxygenase-2 (COX-2). COX-2 is a potent immunological signalling molecule, being part of prostaglandin metabolism and responsible, among other functions, to sensitize MSCs, which have as main and better documented source the bone marrow $[2,3]$. MSCs, after sensitization by COX-2, will deposit themselves above the collagen immature extracellular matrix, generating the granulation tissue [5,6].

As the process continues, some other mediators play their role, as Runt-Related Transcription Factor 2 (RUNX-2) and Osteocalcin (OC). RUNX-2 is a transcription factor that induces MSC differentiation into osteoblasts, which releases OC when mature, 
making Osteocalcin an activity marker for these cells [5-7]. Since the process depends initially on formation of a provisional matrix for later cell signalling and attraction, materials that mimic this matrix are receiving special attention, especially if they can be used as hydrogel, which facilitates its manipulation and application. Putting all of this together, chitosan brings attention among other biomaterials, as it has these characteristics and is also used in tissue engineering [8]. Chitosan is a natural polymer, obtained from partial desacetilation of chitin, which generates a heteropolymer of N-acetyl-D-glucosamine and $\beta-1-4-D$-glucosamines, randomly distributed within the polymer [9].

Previous studies reported that chitosan has low toxicity and allergenicity allied with a good compatibility to biological systems, in vitro and in vivo [10-13]. Additionally, chitosan showed promising results in tissue engineering, as it was used to encapsulate cells aiming tissue regeneration - in hydrogel form, it did not generate any kind of inflammatory response neither it was toxic to cells [14] - and it was also tested as a scaffold for MSCs in cartilage tissue repair, generating benefic results in healing the lesion [15]. Thus, as the material has been shown to be biocompatible and it is possible to use it as a matrix, our aim was to evaluate chitosan capability to be used in tissue engineering in hydrogel form, isolated or loaded with MSCs, evaluating its capability to enhance bone repair.

\section{Materials and Methods}

\section{Hydrogel Synthesis}

The hydrogels were synthesized based on a protocol previously described [16], using commercially available chitosan (448869, Sigma, USA), urease (U1500, Sigma, USA) and urea (U5378, Sigma, USA), in which all of them were previously sterilised by temperature or filtration. The chitosan was dissolved in $\mathrm{HCl} 0,100$ $\mathrm{M}$ at a concentration of $2,5 \%(\mathrm{w} / \mathrm{v})$. Urea was dissolved at two different concentrations - 7,5 $\mathrm{M}$ and $10 \mathrm{M}$ - in ultrapure water. For the hydrogel used in degradation test, the urease was dissolved in phosphate buffer saline (PBS, pH 7,4), meanwhile for the in vitro and in vivo tests, it was dissolved directly in $\alpha$-MEM medium (Vitrocell, Brazil), at the same concentrations - 50, 60 and 70 U/670 $\mu \mathrm{L}$. For the in vitro and in vivo tests, hydrogels were washed every 15 minutes for 1 hour, in order to remove any leaching leftover. The hydrogels had a final volume of $200 \mu \mathrm{L}$.

\section{Degradation Test}

Table 1: Groups of chitosan hydrogels degradation test.

\begin{tabular}{|c|c|c|c|c|}
\hline \multicolumn{2}{|c|}{} & \multicolumn{3}{|c|}{ Units of urease } \\
\hline Time & Urea concentration & Urease 50 U & Urease 60 U & Urease 70 U \\
\hline 21 days & Urea 7,5 M & G1 & G2 & G3 \\
\hline & Urea $10 \mathrm{M}$ & G4 & G5 & G6 \\
\hline
\end{tabular}

The hydrogels were synthesized in different combinations of urea and urease to determine their degradation rate in an environment that simulates the in vivo one. In order to do so, the hydrogels were put in $10 \mathrm{~mL}$ of 1,5 $\mu \mathrm{g} / \mathrm{mL}$ c-type lysozyme (62970, Sigma, USA), which was dissolved in PBS (pH 7,4) and left at $37^{\circ} \mathrm{C}$. The solution was renewed every 3 days. This lysozyme concentration was used to correspond the human serum concentration $[17,18]$. All hydrogels had their initial and final masses measured, with the results being expressed in percentage of initial mass at the end of the experiment. The different hydrogels synthesized are presented in Table 1.

\section{In Vitro Study}

Mesenchymal Stem-Cells Obtainment: The cells used in these experiments were obtained based on a previous protocol [19]. The femur and tibia from a male Wistar rat (4 weeks old) were removed and then immersed in isopropanol 70\%, which were then transferred to PBS 1x. The bones had their ends cut and their medulla washed with $\alpha$-MEM medium (Vitrocell, Brazil) with a $22 \mathrm{G}$ needle (Descarpack, Brazil). The bone extract was centrifuged (5 min, $200 \mathrm{~g}, 4^{\circ} \mathrm{C}$ ) and the pellet resuspended in $\alpha$-MEM medium containing $10 \%$ fetal bovine serum (FBS, Vitrocell, Brazil), which was then transferred to a $75 \mathrm{~cm}^{2}$ flask. Cells were maintained at $37^{\circ} \mathrm{C}$ with a humid atmosphere of $5 \% \mathrm{CO}_{2}$ and the medium was changed every 2 3 days until reach around $80 \%$ confluence.

Mesenchymal Stem-Cells Characterization: In order to characterise the MSCs obtained, we projected to differentiate them in osteocytes and adipocytes, showing their plasticity described initially by Friedenstein et al [1]. All cells induced to differentiation were in third passage. For osteocyte differentiation, we used a specific differentiation medium, mixing StemPro ${ }^{\circledR}$ Osteogenesis Supplement (A10066-01, Gibco ${ }^{\circledR}$, Life Techonologies, USA) with StemPro $^{\circledR}$ Osteocyte/Chondrocyte Differentiation Basal Medium (A10069- 01, Gibco ${ }^{\circledR}$, Life Technologies, USA). In order to differentiate MSCs into adipocytes, it was mixed StemPro ${ }^{\circledR}$ Adipogenesis Supplement (A10065-01, Gibco ${ }^{\circledR}$, Life Technologies, USA) with StemPro ${ }^{\circledR}$ Adipocyte Differentiation Basal Medium (A10410-01, Gibco ${ }^{\circledR}$, Life Technologies, USA). The control groups for both experiments were maintained in $\alpha$-MEM medium (11900, Gibco $^{\circledast}$, Life Technologies, USA) supplemented with 10\% FBS (SH30396.03, HyClone ${ }^{\circledR}$, GE, USA). MSCs were seeded in a 24-well plate (TPP ${ }^{\circledR}$, Sigma, USA). We seeded $10000\left(10^{4}\right)$ cells per well for osteocyte differentiation and $100000\left(10^{5}\right)$ cells per well for adipocyte differentiation, with each well containing $1 \mathrm{~mL}$ of $\alpha$-MEM medium. After 24 hours, we changed half the volume of the medium for $500 \mu \mathrm{L}$ of the specific differentiation medium (in testing group) or $500 \mu \mathrm{L}$ of $\alpha$-MEM (in control group).

This procedure was repeated every 3 4 days. The cells were kept differentiating for 21 days for both differentiation experiments. Control and differentiated groups were prepared in triplicates. After these 3 weeks, groups tested for osteocyte differentiation were fixated for 30 minutes with paraformaldehyde $4 \%$, washed with distilled water and incubated with silver nitrate $5 \%$ in the dark for 30 minutes, being then exposed for 15 minutes to light before observation in optic microscopy. On the other hand, cells subjected to adipocyte differentiation were fixated with paraformaldehyde 
$4 \%$ for 30 minutes, washed with distilled water and incubation in ethanol $70 \%$ for 2 minutes; it was then added $500 \mu \mathrm{L}$ of Sudan II $0,2 \%$ and left for 5 minutes. The Sudan II was then removed and $500 \mu \mathrm{L}$ of distilled water were added to observe the cells under optic microscopy.

Viability Assay: Hydrogels were prepared as described in section 2.1 and transferred to a 24- well plate. Each one was seeded with $100000\left(10^{5}\right)$ cells and received addition $750 \mu \mathrm{L}$ of $\alpha$-MEM medium. The control group wells were seeded with the same number of cells and medium volume, but they did not received the hydrogels. The plate was incubated for 24 hours at $37^{\circ} \mathrm{C} / 5 \%$ $\mathrm{CO}_{2}$. After that, the medium was changed to $\alpha$-MEM containing $10 \%$ alamarBlue ${ }^{\circledR}$ (Thermo Fisher Scientific, USA), using the same volume previously added. The reagent was added to control and test groups. The plate was once again incubated for 24 hours, at $37^{\circ} \mathrm{C} / 5 \% \mathrm{CO}_{2}$. From the supernatant of each well, in duplicate, 200 $\mu \mathrm{L}$ were separated and put in a 96 -well plate (TPP ${ }^{\circledR}$, Sigma, USA) for absorbance reading in a spectrophotometer (Multiskan FC, Thermo Fisher Scientific, USA) at a wavelength of $551 \mathrm{~nm}$.

\section{In Vivo Study}

Experimental Animals: In this study, 30 Wistar male rats (Rattus norvegicus, var. albinus, Rodentia, Mammalia) were used, three months old and initial body weight between 300 and 350 grams. The animals were kept with food and water ad libitum, controlled temperature of $22 \pm 2^{\circ} \mathrm{C}$ and light cycles of 12 hours of light/12 hours of dark. All animals were submitted to surgery in order to produce a critical size bone defect in their calvarium. "Surgeries were performed according to ethical principles of animal care, under standard aseptic conditions and general anaesthesia (experiments approved by Ethics Committee on Animal Use of Federal University of São Carlos (CEUA/UFSCar), under protocol number 8943191015)".

Surgical Procedure: In order to produce the defects in animals' calvarium, a trephine-like dental drill (WMA, Brazil) was used, $2 \mathrm{~cm}$ long and $8 \mathrm{~mm}$ of diameter, activated by a micromotor (Beltec, Brazil), at $13500 \mathrm{rpm}$ and irrigated with normal saline to avoid damage to the bone tissue. Animals were anesthetised with ketamine chloride ( $80 \mathrm{mg} / \mathrm{kg}$, Syntec, Brazil) and xilazine $(10 \mathrm{mg} /$ $\mathrm{kg}$, Syntec, Brazil). Afterwards, when animals were completely anesthetised, their skull was shaved and the area was cleaned with iodine (Rioquímica, Brazil). It was then made an incision of approximately $1.5 \mathrm{~cm}$ in medial region of the scalp, from the nasal bone, in head-tail direction. The lesion was produced keeping the trephine perpendicular to bone surface until exposure of the dura, generating a defect of $8 \mathrm{~mm}$ in diameter. At the end of the surgery, the skin was sutured (Nylon 4-0, Shalon, Brazil) and the local was cleaned. A veterinary antibiotic $(6,000,000 \mathrm{UI}, 20 \mathrm{mg} / 100 \mathrm{~g}$, Zoetis, Brazil) was administered alongside with dipyrone $(0,1 \mathrm{~mL} / 500 \mathrm{~g}$, Dornil, Bravet, Brazil), both in single dose.

Experimental Design: Animals were randomly distributed in three experimental groups ( $\mathrm{n}=10$ per group): Control Group (C), in which the calvarium defect was produced in the animals but they were not treated; Chitosan Group (Ch), in which was applied the chitosan hydrogel into animals' calvarium defect; Chitosan loaded with MSCs Group ( $\mathrm{Ch}+\mathrm{C})$, in which was applied the chitosan hydrogel loaded with MSCs into animals' calvarium defect. All treatments were applied after producing the bone defect. After 21 days of the surgical procedure, the animals were euthanized with a lethal dose of anaesthesia $(285 \mathrm{mg} / \mathrm{kg}$ of ketamine chloride and 36 $\mathrm{mg} / \mathrm{kg}$ of xilazine). Immediately, the skull region where the bone defect was produced was completely removed for further analysis.

\section{Analyses}

Qualitative Histological Analysis: After collection, all the bone samples were fixated in formaldehyde $10 \%$ for 24 hours and washed with water for the next 24 hours; they were then immersed in tetrasodium EDTA $10 \%$ for their decalcification. 60 days later, the samples were removed from EDTA for their histological processing. Samples were embed in paraffin and, from these blocks, it was prepared semi- serial slides of $5 \mu \mathrm{m}$ thick. The slides were stained with haematoxylin-eosin (HE) for further qualitative analysis, through morphological description of bone defects, considering the following criteria: tissue necrosis, presence of inflammatory process, presence of tissue granulation and neoformed bone [20]. Afterwards, all slides were scanned with Pannoramic DESK (3DHISTECH, Hungary) and analysed with Pannoramic VIEWER (3DHISTECH, Hungary), from which we obtained images of the slides.

Morphometric Analysis: From the same blocks obtained previously, semi-serial slides of $5 \mu \mathrm{m}$ thick were prepared, which had the paraffin removed and were rehydrated with alcohol. Then, they were stained with Sirius Red and observed under optic microscope (Axioplan 2, Zeiss, Germany) with a light polariser, which makes collagen fibres vary in colour, from green to red, because of their birefringence. Images were transformed to black and white (black representing the coloured collagen and white the previous black background) and analysed with Image (NIH, USA) (Saraiva et al. [21]) and the total number of pixels in each sample representing total amount of collagen - was counted for posterior comparison between groups.

Immunohistochemical Analysis: This analysis was performed in order to evaluate the expression of the following factors: COX2, RUNX-2 transcription factor and Osteocalcin (OC), all related to osteoblast differentiation and activation [7,22,23]. Starting with the same blocks obtained in item 2.5.1, we prepared semiserial slides $5 \mu \mathrm{m}$ thick, which were incubated with the following primary antibodies at concentration 1:2000: polyclonal antiCOX-2 (Santa Cruz Biotechnology, USA), polyclonal anti-RUNX-2 (Santa Cruz Biotehcnology, USA) and polyclonal anti-OC (Santa Cruz Biotechnology, USA). The slides were first incubated at $4^{\circ} \mathrm{C}$ overnight and then with rabbit anti-IgG secondary antibody (1:200, Vector Laboratories, USA), being followed with avidinbiotin-peroxidase pre-formed complex (Vector Laboratories, USA) and revealed with 3-3'-diaminobenzidine 0,05\%. The slides were then scanned with Pannoramic DESK (3DHISTECH, Hungary) and observed with Pannoramic Viewer (3DHISTECH, Hungary). We evaluated three fields from each slide about presence and quantity of immunostaining, analysing each factor separately. 
Scanning Electron Microscopy (SEM): After collection of the scalps, one sample from each group was separated in order to be observed under SEM. They also passed through the same processes of fixation and decalcification. SEM was performed with Inspect S50 (FEI) from Structural Characterization Laboratory (LCE/DeMA, UFSCar) to acquire images of the lesion areas where biomaterial was implanted, in order to observe bone neoformation.

Statistical Analysis: Data obtained from degradation, in vitro and in vivo tests involving more than two groups were first analysed with Shapiro-Wilk test, in order to evaluate if their distribution is normal. If the distribution is normal, we used ANOVA for comparison of three groups; if distribution was not normal, we used Kruskal-Wallis test. In significant events, it was performed Tukey's test as post-hoc - for ANOVA analyses - or Mann- Whitney - for Kruskal-Wallis analyses, in order to discriminate differences.
In cases of only two groups (as in viability test), T-student test was performed. Analyses were performed in IBM $^{\circledR}$ SPSS $^{\circledR}$ software, all with significance level of $5 \%(p \leq 0,05)$. Graphs were obtained with GraphPad Prism ${ }^{\circledR}$.

\section{Results}

\section{Hydrogel Degradation Test}

The results from degradation test showed there was no significant difference among groups, as data observed generated a $\mathrm{p}$ value of 0,4188 . All groups had a medium degradation rate between $40-60 \%$ in comparison to their initial hydrogel mass; group G5 presented a slightly higher residual mass while G1 a slightly lower final mass, however no statistical significance was observed. The combination chosen for further experiments was $10 \mathrm{M}$ of urea and $50 \mathrm{U}$ of urease/670 $\mu \mathrm{L}$. Data obtained in this test is presented as graphics in Figure 1.

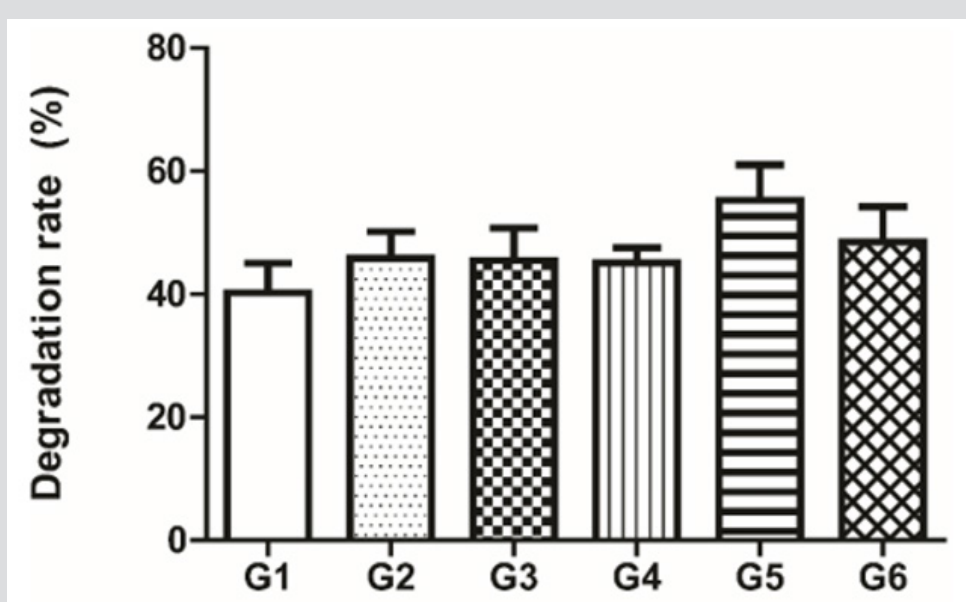

Figure 1: Hydrogel degradation rate of different combinations of urea/urease in hydrogel synthesis: G1: 7,5 M urea and 50 U urease; G2: 7,5 M urea and $60 \mathrm{U}$ urease; G3: 7,5 M urea and $70 \mathrm{U}$ urease; G4: $10 \mathrm{M}$ urea and $50 \mathrm{U}$ urease; G5: $10 \mathrm{M}$ urea and $60 \mathrm{U}$ urease; G6: $10 \mathrm{M}$ urea and $70 \mathrm{U}$ urease. Data is presented as a medium percentage of residual mass in the end of the experiment with respective standard deviation. No statistical difference was observed among groups.

\section{In Vitro Studies}

Viability Test: Results presented in Table 2 shows that average value of absorbance of collected medium from the group that contained hydrogels was $81.67 \%$ in comparison to control group - which was considered as $100 \%$. When T-student test was applied to these values, the $p$-value generated was $p=0,152$, which indicates there was no statistical difference between both groups, demonstrating the cell viability is not compromised when MSCs are put in contact with chitosan hydrogels.

Table 2: Average values of medium absorbance $(551 \mathrm{~nm})$ with alamar Blue ${ }^{\circledR}$, which contained samples either in contact with hydrogels or not.

\begin{tabular}{|c|c|c|}
\hline Group & $\begin{array}{c}\text { Average value } \\
\text { (absorbance) }\end{array}$ & Standard deviation \\
\hline Control & 10,000 & $\pm 0,2185$ \\
\hline Hydrogels & 0,8167 & $\pm 0,1812$ \\
\hline
\end{tabular}

\section{In Vivo Studies}

Qualitative Histological Analysis: Histological analysis did not show, in any group, presence of tissue necrosis. Neoformed bone, on the other hand, was observed in all groups, mostly in marginal areas of the defect. However, it was possible to observe a clear difference between control and both treated groups. In control group, the defect region shows only a thin fibrotic tissue linking the extremities of healthy bone, surrounded in defect edges by granulation tissue and neoformed bone (Figure 2A and 2B).

In the other groups, the region shows a high amount of cells, representing an advanced stage of granulation tissue (Figure 2C-F). There is little difference between groups $\mathrm{Ch}$ and $\mathrm{Ch}+\mathrm{C}$ : in comparison to the first group, the latter presents a slightly higher amount of granulation tissue, which is observed mostly in the centre of the defect. Thus, there is an indication that hydrogel presence - whether it is loaded with cells or not - helped the bone repair process. In treated groups, there is also a reddish region inside the defect, indicated by asterisks in Figures 2D and 2F. In group $\mathrm{Ch}$, there are no cells inside this area (Figure 2C and 2D); in group $\mathrm{Ch}+\mathrm{C}$, which chitosan was loaded with MSCs, however, the amount of cells is much higher in this region (represented by arrows in Figure 2F), indicating this reddish area in treated groups is a residual biomaterial in defect area (Figure $2 \mathrm{E}$ and $2 \mathrm{~F}$ ). 


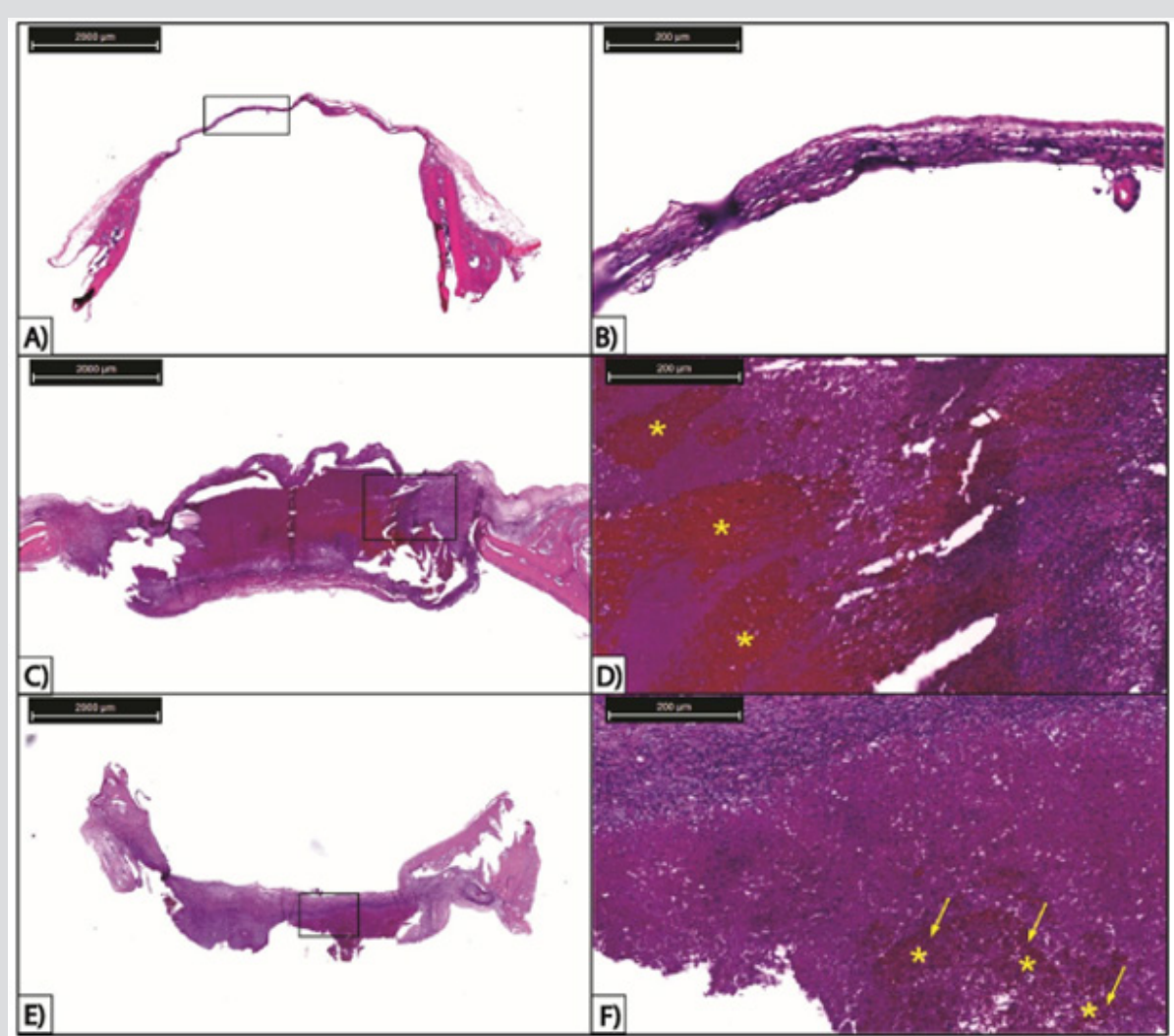

Figure 2: Observation of histological sections of three experimental groups stained with haematoxylin-eosin. A) Control group. Scale bar of $2000 \mu \mathrm{m}$. B) Fibrotic tissue section from control group. Scale bar of $200 \mu \mathrm{m}$. C) Group treated with isolated chitosan. Scale bar of $2000 \mu \mathrm{m}$. D) Region within defect area of group treated with isolated chitosan. Asterisks indicate reddish region of residual chitosan with absence of cells. Scale bar of $200 \mu \mathrm{m}$. E) Group treated with mesenchymal stem cell-loaded chitosan. Scale bar of $2000 \mu \mathrm{m}$. F) Closer observation of a region within defect area of group treated with mesenchymal stem cell-loaded chitosan. Asterisks indicate reddish region of residual chitosan within defect area and arrows indicate cells inside this region. Scale bar of $200 \mu \mathrm{m}$.

Morphometric Analysis: The analysis of amount of collagen in samples did not showed statistical difference in comparison between control and MSC-loaded chitosan groups and between isolated chitosan and MSC-loaded chitosan groups. However, the group treated with isolated chitosan showed a higher presence, statistically significant ( $p=0,0066$ ), of collagen in comparison to control group, as it can be observed in Figure 3.

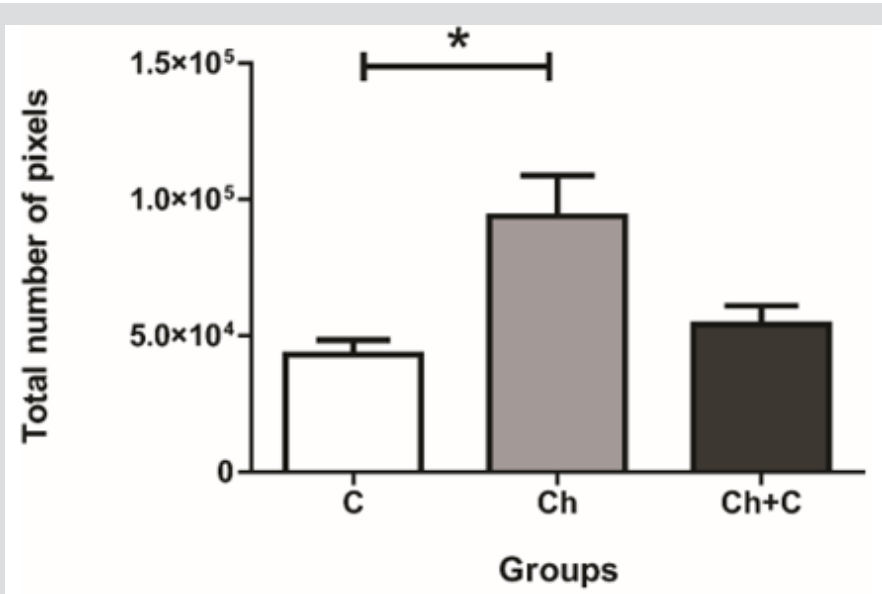

Figure 3: Statistical comparison of total amount of collagen observed in all samples from each group (C: control group, no treatment applied; $\mathrm{Ch}$ : group treated with isolated chitosan hydrogel; $\mathrm{Ch}+\mathrm{C}$ : group treated with mesenchymal stem cellloaded chitosan hydrogel. Groups are indicated and asterisk represent statistically significant difference. 
Immunohistochemical Analysis: Samples marked with antibodies anti-RUNX-2 and anti-OC showed immunostaining as much in cells as in extracellular matrix. However, when statistically compared, there was no significant variation among control and treated groups. Immunostaining with anti-COX-2 also marked cells and ECM alike but, differently from other two markers, it had a significant difference between group $\mathrm{Ch}$ and group $\mathrm{C}(\mathrm{p}=0,0268)$. There was no difference in comparisons between group $\mathrm{Ch}$ and group $\mathrm{Ch}+\mathrm{C}$ and between group $\mathrm{C}$ and group $\mathrm{Ch}+\mathrm{C}$. Results can be observed in Figures 4, 5 and 6.

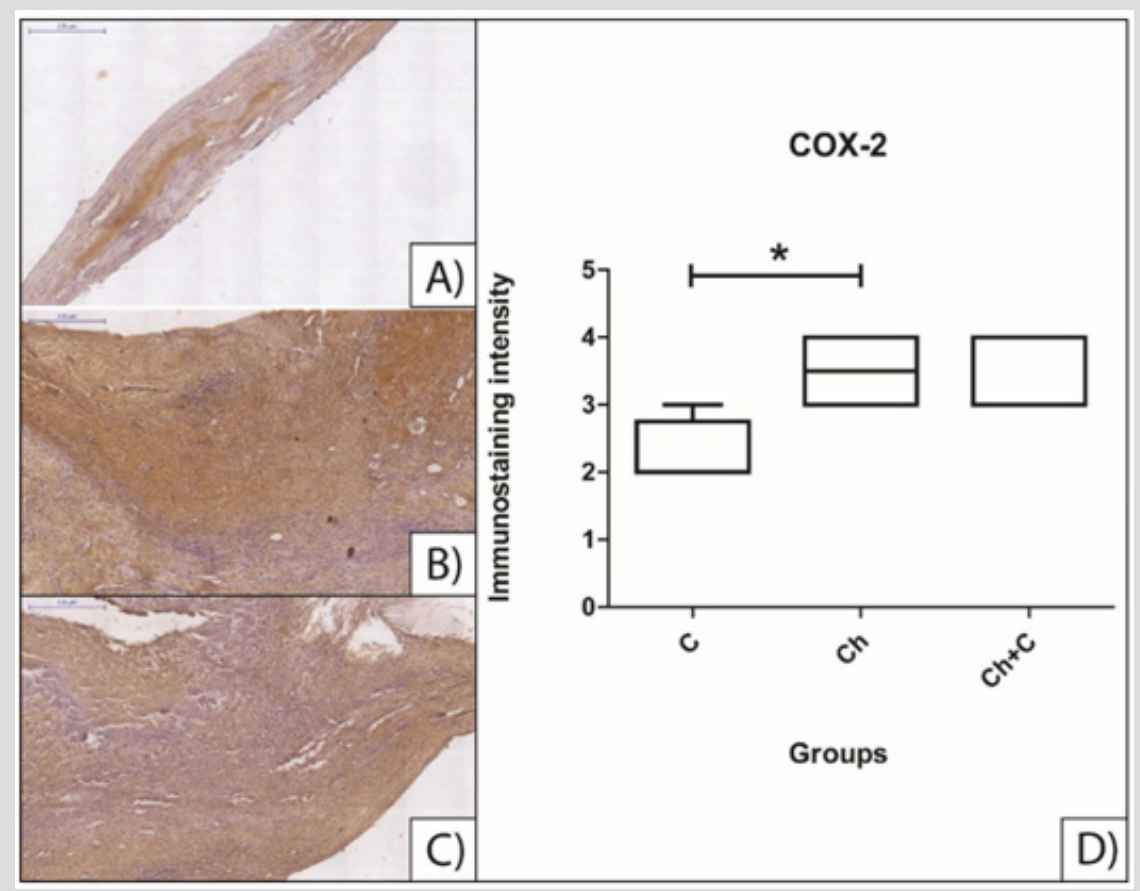

Figure 4: Immunohistochemical analysis of slides from calvarial bone immunostained with COX-2. A) group C, no treatment; B) group $\mathrm{Ch}$, treated with isolated chitosan hydrogel; C) group $\mathrm{Ch}+\mathrm{C}$, treated with mesenchymal stem cell-loaded chitosan hydrogel; D) statistical comparison between three groups. Asterisk indicate significant difference. All scale bars are $200 \mu \mathrm{m}$.

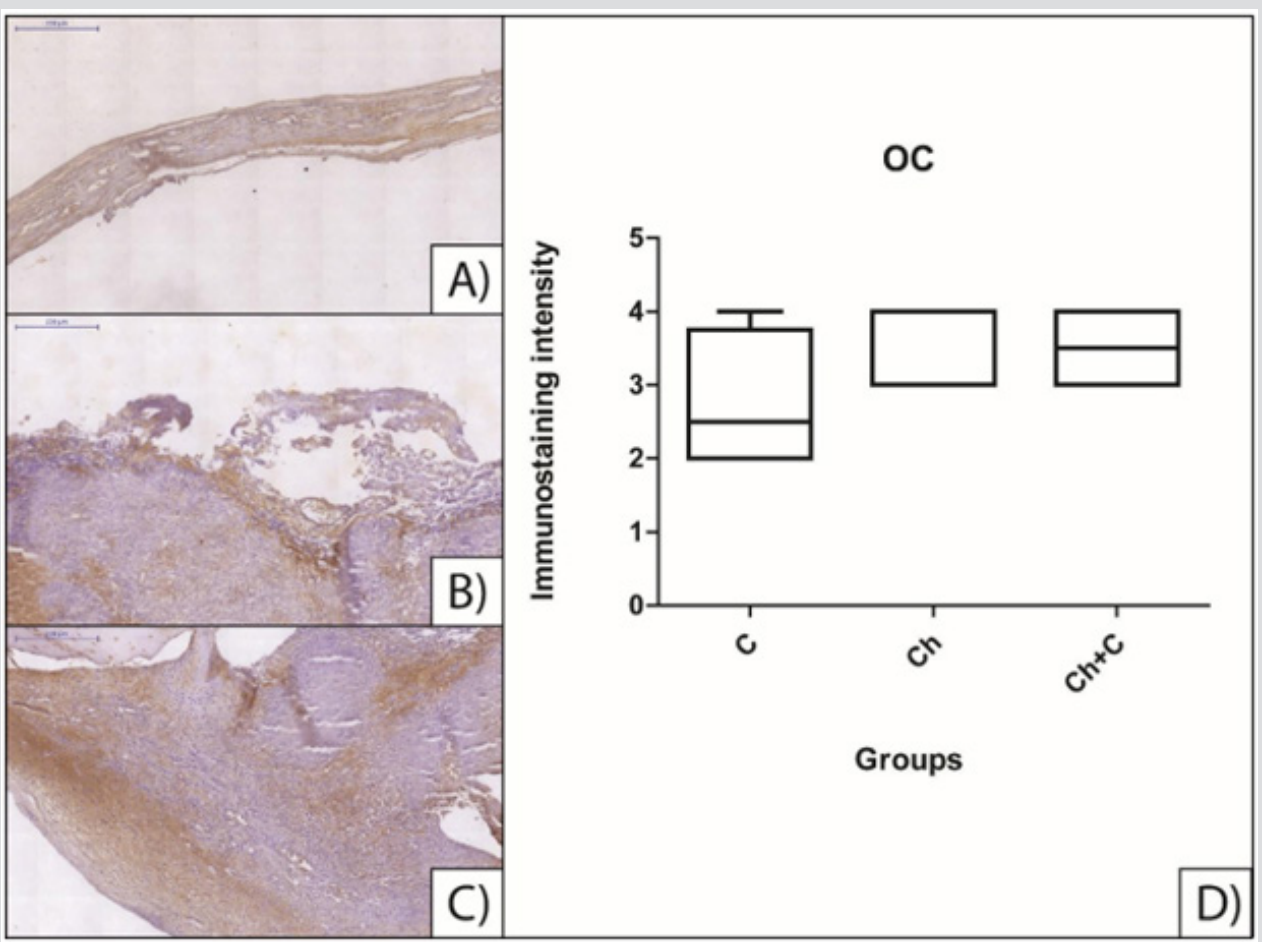

Figure 5: Immunohistochemical analysis of slides from calvarial bone immunostained with OC. A) group C, no treatment; B) group $\mathrm{Ch}$, treated with isolated chitosan hydrogel; C) group $\mathrm{Ch}+\mathrm{C}$, treated with mesenchymal stem cell-loaded chitosan hydrogel; D) statistical comparison between three groups. All scale bars are $200 \mu \mathrm{m}$. 


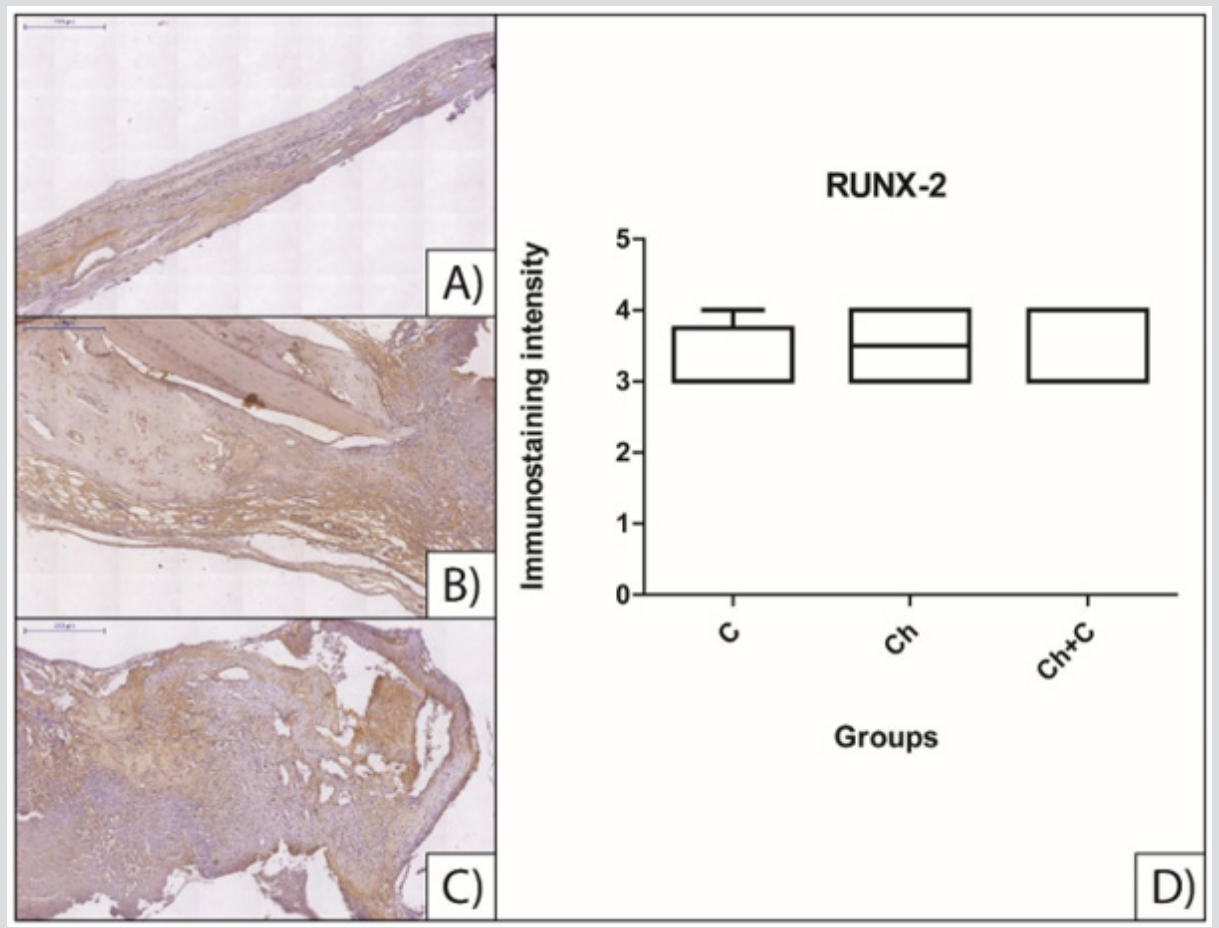

Figure 6: Immunohistochemical analysis of slides from calvarial bone immunostained with RUNX-2. A) group C, no treatment; B) group $\mathrm{Ch}$, treated with isolated chitosan hydrogel; C) group $\mathrm{Ch}+\mathrm{C}$, treated with mesenchymal stem cell-loaded chitosan hydrogel; D) statistical comparison between three groups. All scale bars are $200 \mu \mathrm{m}$.

Scanning Electron Microscopy (SEM): Results obtained by SEM are presented in Figure 7. Images show the sample density - less dense the sample, darker the image. Thus, the presence of darker regions within the defect in Figures 7A, which represents the control group, indicate that the tissue inside the lesion does not have the same density of the tissue outside defect area, consequently showing the absence of mineralised bone tissue. In addition, it is possible to observe the defect edge with no integration. In the images representing treated groups (Figure 7B for group Ch; Figure 7C for group $\mathrm{Ch}+\mathrm{C}$ ), there is no exact delimitation of the lesion edge, which we understand to occur due to the treatment with the biomaterial. Still, in the same figures, there are no dark regions indicating a less dense tissue in comparison to pre-existing bone, which suggests the region within the lesion is a neoformed bone. In the images, asterisks indicate the region of original and healthy bone, while arrows present the edge of the lesion.

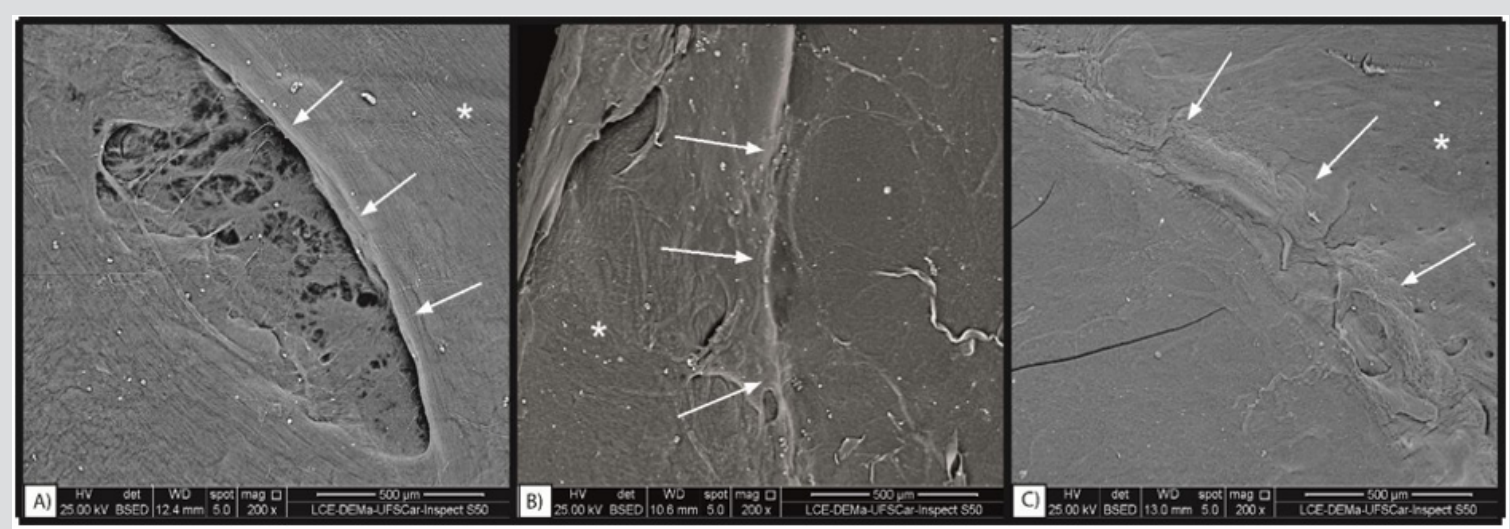

Figure 7: Results from Scanning Electron Microscopy (SEM) of the scalp samples. A) Control group; arrows indicate the lesion border and asterisk indicates the healthy bone. B) Group treated with isolated chitosan; arrows indicate lesion border and asterisk indicates preformed bone. C) Group treated with chitosan loaded with mesenchymal stem cells; arrows indicate lesion border and asterisk indicates healthy bone region outside the lesion. All scale bars are $500 \mu \mathrm{m}$.

\section{Discussion}

The study presented had as objective to observe the efficacy of a chitosan hydrogel, associated or not to MSCs, in repair a critical size bone defect produced in rat calvaria. As the experiments were performed, it was possible to observe the hydrogel effects in vitro as much as in vivo, from cellular viability analysis when in contact with the material to histological analysis of the calvaria. The results obtained in hydrogel synthesis show similar patterns in comparison to what is reported in literature. Yan et al. [16] 
showed that chitosan hydrogel gelation is possible to occur using urea and urease in urea concentrations as low as $30 \mathrm{mM}$. The same research shows that higher concentrations of both reagents make hydrogels to gelate faster; also, higher urease concentrations cause hydrogels to degrade in a higher rate in comparison to those with lower concentrations. A second report conducted by Wlodarczyk et al. [24] showed the kinetics of chitosan hydrogel gelation and observed the increase in $\mathrm{pH}$ that causes chitosan to become a hydrogel, while it is aqueous in lower values of $\mathrm{pH}$ - they, however, do not observe differences in hydrogel degradation rates according to variances in urea/urease concentrations.

Wlodarczyk et al. [24] observed higher concentrations of urease caused hydrogel to gelate faster, as it achieves the basal $\mathrm{pH}$ for gelation; they also show higher concentrations of urea cause hydrogel to gelate faster until it reaches a certain concentration which beyond it does not interfere; maximum concentrations used in these experiments were $150 \mathrm{mM}$ of urea and $10,5 \mathrm{U} / \mathrm{mL}$ of urease. In our study, in order to obtain gelation, urea concentrations used in our experiments were 7,5 $\mathrm{M}$ and $10 \mathrm{M}$, with a minimum urease of $50 \mathrm{U}$. Moreover, it is not possible to observe here statistical differences in hydrogel degradation between different combination of urea and urease concentrations. Our study focused on synthesizing a material that does not degrade rapidly, working as an initial support for development of repair process, but also does not last long periods of time, giving place to neoformed tissue; hence, the chosen combination was urea $10 \mathrm{M}$ and urease $50 \mathrm{U}$.

Passing on to histological results, they showed there was no tissue necrosis in any group and also, there is bone neoformation in all groups with a clear difference between control and treated groups. Control group exhibit only small regions of neoformed bone around lesion edges with fibrotic tissue linking these regions; the presence of fibrotic tissue is due to the fact it is a critical size defect, meaning there is no spontaneous regeneration of the tissue [25]. On the other hand, treated groups $(\mathrm{Ch}$ and $\mathrm{Ch}+\mathrm{C})$ showed a great number of cells within the defect regions, while also exhibited neoformed bone in marginal regions of the lesion. This cell agglomeration is typical of granulation tissue, characterised by a great cellular population, which among them there are several proliferative fibroblasts, responsible for synthesizing the extracellular matrix starting from collagen deposition, which will later be substituted by neoformed bone. The granulation tissue is more commonly observed when the acute inflammatory stage of bone repair is diminished, which lasts for about 7 days, reinforcing our observation that this cellular agglomerate is the granulation tissue $[6,26]$. Apart from the presence of the granulation tissue, we noted the absence of neoformed bone across the whole defect region, which we understand that occurs due to short experimental time we applied in our tests. A previous study [27] reported that, in the same model of bone defect of our study, it was necessary 8 weeks for the defect to be almost completely repaired when it was subjected to a treatment, while Du et al. [28] observed that this time of 8 weeks was enough for complete repair with a specific treatment. Lohmann et al. [29] showed that only with 7 weeks of treatment it was possible to observe a neoformed bone that covered, almost completely, the defect region. Thus, our results present the suggestion that an experimental period of 3 weeks is too short to observe bone neoformation in this model of critical size defect in rats, which maybe would be different if a longer period of treatment was applied.

Another difference observed among groups in histological results is the presence of a reddish region in the interior of defect area in groups treated with the hydrogel, either loaded or not with MSCs. Meanwhile, the same pattern is not observed in control group. This region in groups $\mathrm{Ch}$ and $\mathrm{Ch}+\mathrm{C}$ is caused by the residual biomaterial still present in the defect, which agrees to the data reported by Cui et al. [30], who also observed, after staining with haematoxylin-eosin, a reddish region due to the chitosan. This result in histological analysis corroborates with our degradation test result, showing that, in 3 weeks, the hydrogel does not completely degrade, neither in vitro nor in vivo. The presence of cells in this region only in group $\mathrm{Ch}+\mathrm{C}$ also shows an agreement to what was observed in viability test, showing cells still remain alive inside hydrogels.

Results obtained from morphometric analysis were also interesting. Firstly, Junqueira et al. [31] demonstrated that combination of Sirius Red staining to optic microscopy with light polariser has a high specificity to collagen marking, given that collagen fibres have high birefringence when in association to this stain, causing them to be observed coloured. Thus, our work shows the group treated with isolated chitosan has a higher amount of collagen inside defect region when compared to other two. As collagen deposition is one of the first steps in bone repair [6], the great amount of collagen in group $\mathrm{Ch}$ is the immature extracellular matrix, which will later be calcified,unlike control group, in which the collagen observed is only result of the formation of fibrous tissue between defect extremities [6,25,32].

Immunohistochemical assays showed there was no significant difference between groups when analysed antigens RUNX-2 and OC; however, a difference was observed when anti-COX-2 was used for immunostaining, as group $\mathrm{Ch}$ had a significant higher expression in comparison to control group. Literature reports RUNX-2 as a transcription factor closely related to bone formation, being associated to osteoblast differentiation and expressed in this type of cell to act as a control for expression of several factors related to matrix deposition in bone, including Osteocalcin [33-35]. OC, on the other hand, is the non-collagenous protein with higher abundance in the bone; it is synthesized specifically and uniquely by osteoblasts and it is found at high concentrations in mineral matrix, which makes it a very used and important marker for bone formation [3638]. Finally, COX-2 is an important inflammatory mediator activated in prostaglandin metabolism, with an essential role in preliminary stages of bone repair, and also acting as an activator of osteoblastic differentiation [23]. These results agree with histological data and indicate, once more, that, in this experimental period we used in our study, it is not possible to observe definitive role of osteoblasts in bone defect - since their activity markers are not differentially expressed -, but possible to identify stages of bone repair, in group $\mathrm{Ch}$, in which the role of COX-2 are extremely important, as induction of differentiation of Mesenchymal Stem Cells into osteoblasts [23]. 
In conclusion, our study shows that chitosan, as a hydrogel, has a great capacity to assist bone defect repair, even though it is not loaded with Mesenchymal Stem Cells. Our results demonstrate that although 3 weeks may be a short experimental period, the hydrogel showed itself as a promising option, as it allows evolution of bone repair process, demonstrating chitosan as an important biomaterial for tissue engineering and regenerative medicine. As a suggestion for future study, the investigation of chitosan's potential for fracture healing, which may turn it an option for future clinical applications.

\section{Acknowledgment}

We would like to thank Fundação de Amparo à Pesquisa do Estado de São Paulo (FAPESP) for the financial support as the scholarship number 2015/23632-8.

\section{References}

1. Friedenstein AJ, Piatetzky Shapiro II, Petrakova KV (1966) Osteogenesis in transplants of bone marrow cells. J Embryol Exp Morphol 16(3): 381390.

2. Fellows CR, Matta C, Zakany R, Khan IM, Mobasheri A (2016) Adipose, Bone Marrow and Synovial Joint-Derived Mesenchymal Stem Cells for Cartilage Repair. Front Genet 7: 213.

3. Park JS, Suryaprakash S, Lao YH, Leong KW (2015) Engineering mesenchymal stem cells for regenerative medicine and drug delivery. Methods Elsevier Inc 84: 3-16.

4. Bielby R, Jones E, McGonagle D (2007) The role of mesenchymal stem cells in maintenance and repair of bone. Injury 38(Suppl 1): S26-S32.

5. Giannoudis P V, Jones E, Einhorn TA (2011) Fracture healing and bone repair. Injury 42(6): 549-550.

6. Loi F, Córdova LA, Pajarinen J, Lin T, Yao Z, et al. (2016) Inflammation, fracture and bone repair. Bone. Elsevier 86: 119-130.

7. Tim CR, Bossini PS, Kido HW, Malavazi I, von Zeska Kress MR, et al. (2015) Effects of low-level laser therapy on the expression of osteogenic genes during the initial stages of bone healing in rats: a microarray analysis. Lasers Med Sci 30(9): 2325-2333.

8. Lewandowska Łańcucka J, Fiejdasz S, Rodzik Ł, Łatkiewicz A, Nowakowska M (2015) Novel hybrid materials for preparation of bone tissue engineering scaffolds. J Mater Sci Mater Med 26(9): 231.

9. Cheung RCF, Ng TB, Wong JH, Chan WY (2015) Chitosan: An update on potential biomedical and pharmaceutical applications. Mar Drugs 13(8): 5156-5186.

10. Kumar RMN V, Muzzarelli RAA, Sashiwa H, Domb AJ (2004) Chitosan chemistry and phamaceutical perspective. Chem Rev 104(12):60176084

11. Asthana S, Goyal P, Dhar R, KU, Pampanaboina NB, Christakiran J, et al. (2015) Evaluation extracellular matrix-chitosan composite films for wound healing application. J Mater Sci Mater Med 26(8): 220.

12. Upadhyay J, Kumar A, Gupta K, Mandal M (2015) Investigation of physical and biological properties of polypyrrole nanotubes - chitosan nanocomposites. Carbohydr Polym 132: 481-489.

13. Kaderli S, Boulocher C, Pillet E, Watrelot Virieux D, Rougemont AL, et al. (2015) A novel biocompatible hyaluronic acid-chitosan hybrid hydrogel for osteoarthrosis therapy. Int J Pharm 483(1-2): 158-168.

14. Yan XZ, van den Beucken JJJP, Cai X, Yu N, Jansen JA, et al. (2015) Periodontal Tissue Regeneration Using Enzymatically Solidified Chitosan Hydrogels With or Without Cell Loading. Tissue Eng. Part A 21(5-6): 1066-1076.

15. Azarpira MR, Shahcheraghi GH, Ayatollahi M, Geramizadeh B (2015) Tissue engineering strategy using mesenchymal stem cell-based chitosan scafolds in growth plate surgery: A preliminary study in rabbits. Orthop Traumatol Surg Res 101(5): 601-605.

16. Yan XZ, Nijhuis AWG, Van Den Beucken JJJP, Both SK, Jansen JA, et al (2014) Enzymatic control of chitosan gelation for delivery of periodontal ligament cells. Macromol Biosci 14(7): 1004-1014.

17. Freier T, Koh HS, Kazazian K, Shoichet MS (2005) Controlling cell adhesion and degradation of chitosan films by $\mathrm{N}$-acetylation. Biomaterials 26(29): 5872-5878.

18. Masuda T, Ueno Y, Kitabatake N (2001) Sweetness and enzymatic activity of lysozyme. J Agric Food Chem 49(10): 4937-4941.

19. Zhang L, Chan C (2010) Isolation and enrichment of rat mesenchymal stem cells (MSCs) and separation of single-colony derived MSCs. J Vis Exp 37: 1852.

20. Tim CR, Bossini PS, Kido HW, Malavazi I, Von Zeska Kress MR, Carazzolle $\mathrm{MF}$, et al. (2016) Effects of low level laser therapy on inflammatory and angiogenic gene expression during the process of bone healing: A microarray analysis. J Photochem Photobiol B Biol 154: 8-15.

21. Saraiva JA, Fonseca TS, Silva GF, Sasso Cerri E, Guerreiro Tanomaru JM, Tanomaru Filho M, et al. (2018) Reduced interleukin-6 immuno expression and birefringent collagen formation indicate that MTA Plus and MTA Fillapex are biocompatible. Biomed Mater 13(3): 035002.

22. Kang Q, Sun MH, Cheng H, Peng Y, Montag AG, et al. (2004) Characterization of the distinct orthotopic bone-forming activity of 14 BMPs using recombinant adenovirus-mediated gene delivery. Gene Ther 11(17): 1312-1320.

23. Zhang X, Schwarz EM, Young DA, Edward Puzas J, Rosier RN, et al. (2002) Cyclooxygenase- 2 regulates mesenchymal cell differentiation into the osteoblast lineage and is critically involved in bone repair. J Clin Invest 109(11): 1405-1415.

24. Wlodarczyk D, Méricq JP, Soussan L, Bouyer D, Faur C (2017) Enzymatic gelation to prepare chitosan gels: Study of gelation kinetics and identification of limiting parameters for controlled gel morphology. Int ] Biol Macromol 107(Pt A): 1175-1183.

25. Hollinger JO, Kleinschmidt JC (1990) The Critical Size Defect as an experimental Model To Test Bone Repair Materials. J Craniofac Surg 1(1): 60-68.

26. Vieira AE, Repeke CE, De Barros Ferreira S, Colavite PM, Biguetti CC, et al. (2015) Intramembranous bone healing process subsequent to tooth extraction in mice: Micro-computed tomography, histomorphometric and molecular characterization. PLoS One 10(5): 1-22.

27. Jang JW, Lee JS, Jung UW, Kim CS, Cho KS (2016) In vivo evaluation of commercially available gel-type polyethylene glycol membrane for carrier of recombinant human bone morphogenetic protein-2. J Oral Maxillofac Surg 75(2): 297-313

28. Du M, Zhu T, Duan X, Ge S, Li N, et al. (2017) Acellular dermal matrix loading with bFGF achieves similar acceleration of bone regeneration to BMP-2 via differential effects on recruitment, proliferation and sustained osteodifferentiation of mesenchymal stem cells. Mater Sci Eng C Mater Biol Appl 70(Pt 1): 62-70.

29. Lohmann P, Willuweit A, Neffe AT, Geisler S, Gebauer TP, Beer S, et al. (2016) Bone regeneration induced by a 3D architectured hydrogel in a rat critical-size calvarial defect. Biomaterials 113: 158-169.

30. Cui J, Liang J, Wen Y, Sun X, Li T, et al. (2014) In vitro and in vivo evaluation of chitosan/ $\beta$-glycerol phosphate composite membrane for guided bone regeneration. J Biomed Mater Res 102(9): 2911-2917.

31. Junqueira LCU, Bignolas G, Brentani RR (1979) Picrosirius staining plus polarization microscopy, a specific method for collagen detection in tissue sections. Histochem J 11(4): 447-455.

32. Takagi K, Urist MR (1982) The reaction of the dura to bone morphogenetic protein (BMP) in repair of skull defects. Ann Surg 196(1): 100-109. 
33. Saler M, Caliogna L, Botta L, Benazzo F, Riva F, et al. (2017) hASC and DFAT, Multipotent Stem Cells for Regenerative Medicine: A Comparison of Their Potential Differentiation In Vitro. Int. J Mol Sci 18(12): 2699.

34. Tai PWL, Wu H, Van Wijnen AJ, Stein GS, Stein JL, et al. (2017) Genomewide DNase hypersensitivity, and occupancy of RUNX2 and CTCF reveal a highly dynamic gene regulome during MC3T3 pre-osteoblast differentiation. PLoS One 12: 1-23.

35. Son HE, Kim EJ, Jang WG (2017) Curcumin induces osteoblast differentiation through mild-endoplasmic reticulum stress-mediated such as BMP2 on osteoblast cells. Life Sci 193: 34-39.

\section{ISSN: 2574-1241}

DOI: 10.26717/BJSTR.2019.17.002986

Carla Roberta Tim. Biomed J Sci \& Tech Res

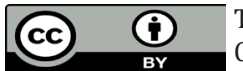

This work is licensed under Creative Commons Attribution 4.0 License

Submission Link: https://biomedres.us/submit-manuscript.php
36. Brennan Speranza TC, Conigrave AD (2015) Osteocalcin: an osteoblastderived polypeptide hormone that modulates whole body energy metabolism. Calcif Tissue Int 96(1): 1-10.

37. Ferron M, Lacombe J (2014) Regulation of energy metabolism by the skeleton: Osteocalcin and beyond. Arch Biochem Biophys 561: 137-146.

38. Wei J, Karsenty G (2015) An overview of the metabolic functions of osteocalcin. Rev Endocr Metab Disord 16(2): 93-98.

$\begin{array}{ll}\text { BIOMEDICAL } & \text { Assets of Publishing with us } \\ \text { RESEARCHES } & \text { - Global archiving of articles } \\ \text { - Immediate, unrestricted online access } & \text { - Rigorous Peer Review Process } \\ & \text { - Authors Retain Copyrights }\end{array}$

